\title{
COMPUTATIONAL HYDRODYNAMIC SIMULATIONS FOR AN UNDERWATER AXISYMMETRIC HEMISPHERE-CYLINDER HULL-FORM AT INCIDENCE
}

\author{
Salimuddin Zahir*
}

Centres of Excellence in Science \& Applied Technologies, Islamabad - 44000, Pakistan, E-mail: cfdpak@apollo.net.pk

\begin{abstract}
:
This contribution presents computational hydrodynamic simulation results for a generic hull shape with an attached short pin-protuberance. This work is a part of a large framework of numerical simulation and experimentation carried out for blunted head-forms of various hemispheric shapes, for determination of aero-hydrodynamic coefficients and their static stability features. Presently results of static axial and circumferential pressure distribution on the surface are presented for a clean hull-form with the pin-protuberance, calculations are made for a selected velocity with varying angle of attacks, under a non-cavitating depth. It is shown that a suitably located short lateral pin has an adequate effectiveness to control pitch maneuver of an underwater hemispherecylinder hull-form. A suitable pin-height deployment commensurate to required pitch attitude trajectory correction is found to be a workable idea. Simulation results show that the concept has potential of effective pitch attitude control of the hemisphere-cylinder hull-form.
\end{abstract}

Keywords: Autonomous underwater vehicles, hemisphere-cylinder hull-form, axisymmetric hydrodynamic configuration, axial pressure distribution, CFD, hydrodynamic force coefficients, static stability.

\section{Nomenclature:}

\begin{tabular}{|c|c|}
\hline V & Freestream velocity $[\mathrm{m} / \mathrm{sec}]$ \\
\hline$p_{\infty}$ & Freestream pressure $\left[\mathrm{N} / \mathrm{m}^{2}\right]$ \\
\hline $\mathrm{Re}$ & $\begin{array}{l}\text { Reynolds number based on diameter } \\
\text { of cylinder }\end{array}$ \\
\hline$\Phi$ & Circumferential angel [deg] \\
\hline$C_{p}$ & Pressure coefficient \\
\hline $\mathrm{p}$ & Local static pressure $[\mathrm{Pa}]$ \\
\hline$\alpha$, AoA & Angle-of-attack [degree] \\
\hline$T_{\infty}$ & Reference temperature $[\mathrm{K}]$ \\
\hline$P_{\text {inf }}$ & Reference pressure $[\mathrm{Pa}]$ \\
\hline$\rho_{\infty}$ & Reference fluid density $\left[\mathrm{kg} / \mathrm{m}^{3}\right]$ \\
\hline $\mathrm{R}$ & Nose, cylinder body radius [m] \\
\hline M & Mach number \\
\hline H, D & Pin height/ diameter [m] \\
\hline$\rho_{\mathrm{m}}$ & Mixture density $\left[\mathrm{kg} / \mathrm{m}^{3}\right]$ \\
\hline$\alpha_{k}$ & Volume fraction of phase $\mathrm{k}$ \\
\hline$\dot{m}$ & Mass transfer due to cavitation $[\mathrm{kg} / \mathrm{s}]$ \\
\hline $\bar{v}_{d r, k}$ & Drift velocity of secondary phase $[\mathrm{m} / \mathrm{s}]$ \\
\hline $\mathrm{R}, \mathrm{r}$ & Bubble radius $[\mathrm{m}]$ \\
\hline$p_{B}$ & Pressure inside bubble $\left[\mathrm{N} / \mathrm{m}^{2}\right]$ \\
\hline$p_{v}$ & Vapor pressure $\left[\mathrm{N} / \mathrm{m}^{2}\right]$ \\
\hline$\varphi$ & Volume of individual bubble $\left[\mathrm{m}^{3}\right]$ \\
\hline$\eta$ & $\begin{array}{l}\text { Number of bubbles per unit volume of } \\
\text { liquid }\left[1 / \mathrm{m}^{3}\right]\end{array}$ \\
\hline$y^{+}$ & Non-dimensional wall distance \\
\hline$C_{m}$ & Pitching moment coefficient \\
\hline$X_{C p} / L$ & Non-dimensional centre of pressure \\
\hline
\end{tabular}

$\begin{array}{ll}C_{p} & \text { Non-dimensional pressure coefficient } \\ C_{Y} & \text { Normal force coefficient } \\ C_{Y_{\alpha}} & \text { Normal force derivative } \\ C_{m_{\alpha}} & \text { Pitching moment derivative } \\ L & \text { Length of the cylindrical body [m] } \\ q_{\infty} & \text { Non-dimensional free stream } \\ & \text { dynamic pressure } \\ \mathrm{P}_{\mathrm{P}} \mathrm{P}_{\mathrm{inf}}, K & \text { Non-dimensional pressure ratio } \\ C_{X} & \text { Axial drag force coefficient } \\ \mathrm{p}_{\Re} & \left.\text { Free stream pressure [N/m } / \mathrm{m}^{2}\right] \\ \mathrm{V}_{\Re} & \text { Free stream velocity [m/s] } \\ \sigma & \text { Cavitation number } \\ \mu & \left.\text { Dynamic viscosity of fluid [N.s } / \mathrm{m}^{2}\right] \\ \vec{v}_{m} & \text { Mass averaged velocity [m/s] } \\ \mathrm{q}_{\infty} & \text { Free Stream Dynamic Pressure, psi or } \\ & \text { psf } \\ \mathrm{x}, \mathrm{y} & \text { Coordinate system axis } \\ \mathrm{x} / \mathrm{L} & \text { Non-dimensional axial length }\end{array}$

\section{Subscripts}

$\infty \quad$ Free stream conditions

$x \quad$ Streamwise coordinates

$\mathrm{k} \quad$ Primary phase

p Secondary phase

v Physical quantities of vapour 


\section{Introduction}

Submersible systems categorized as autonomous underwater vehicles (AUV) and remotely operated vehicles (ROV) are unmanned vehicles applied for inspection, surveys and precision maneuvering for complex operations and applications. Generally simple applications involve, slow speeds, small pitch angles and low turn rates, suitable to mission requirements. Although complexity may arise when the mission is supplemented by operations in hazardous environment requiring precise autopilot functionality for safe maneuvers, that may give rise to complex flow situation over an AUV hull-form. Such a flow situation over blunt nosed hull shape geometry is characterized with flow separation and formation of complex flow structures. The resultant flowfields pose difficulties in simulation for capturing accurate turbulent free shear layers and prediction of transition to turbulence. Hence, simulation of the flow past finite axisymmetric blunt nose bodies is of considerable importance in both naval engineering and underwater hydrodynamics.

The present investigations sets out to address the aspect of introducing a short pin-protuberance on a hemisphere-cylinder head-form, there by aiming to investigate the changes in hydrodynamic force coefficients, i.e., normal, axial force and pitching moment coefficients acting over the hull-form with pin underwater at various angles of attack and speed. Earlier most of the experimental investigations on blunt nosed axisymmetric underwater bodies have been conducted in wind tunnel by Granville (1953), Wallskog et al (1953), Polhamus (1957), Ward (1978), Liu et al (1995) and Hackett (2000) at different Reynolds number and by Hsieh (1977) and Ngoc (1999) for bodies at incidence. Configurations at various depths of submergence from the free surface were studied by Nakayama et al (1974) and Roddy (1990) in a towing tank. Some investigators like Li et al (2010) also measured drag and added mass coefficients. Though, hydrodynamic coefficients derived from the towing tank investigations are limited in literature, more recent approach is through an application of computational fluid dynamics (CFD) techniques. Kunz et al (1999) and Jagadeesh et al (2005) had investigated various issues related to the application of CFD to various aspects of underwater hydrodynamics, including, selection of turbulence models, grid generation and boundary resolution techniques, while cavitation and bubble dynamics was studied by Plesset et al (1971), Sung et al and Merkle et al (1998) mostly for the axisymmetric bodies. While some recent AUV hull-form studies are carried by Zahid et al (2002), Alvarez et al (2009), Xianzhao et al (2010) and Aloota et al (2011). The present approach considers simulation of hydrodynamic characteristics on a head-form of a typical autonomous underwater vehicle (AUV) under normal operating conditions and further its hydrodynamic pitch maneuvering control is investigated with introduction of an extendable short pin over its leeward cylindrical surface. The computed force coefficients are compared in a speed range and for various angles of attack. The angles of attack considered are in a range of zero to -12 degrees. The study suggest that the alteration of flow symmetry cause a net normal force, usable in creation of a desired pitching moment and it very significantly as a function of angle of attack, where only negative incidence angles under a nose-down maneuver demonstrate the potential for the use of short-pin for hull-form maneuver control.

In this study, baseline model with theoretical formulation of the computational method, including physical model details is discussed. Systematic validation results for a zero-incidence drag computations at fixed velocity and for an axisymmetric 0.5 caliber axial pressure distribution got compared with experimental data at a range of cavitation numbers is shown. In this present paper, stability characteristics of a hull-form with extendable pin-height is demonstrated by analyzing different Reynold's number about a hemisphere-cylindrical geometry with a hemispherical fore-body at several angles of attack. In addition, pin effects were highlighted through computation of circumferential pressure distribution in the pin aft and cavitation effects were also shown at the nose-cylinder interface.

\section{Computational Methodology}

\subsection{Model geometry}

Aero-hydrodynamic model considered in present study is a head-form with an axisymmetric hemispherical nose attached to an aft cylinder.

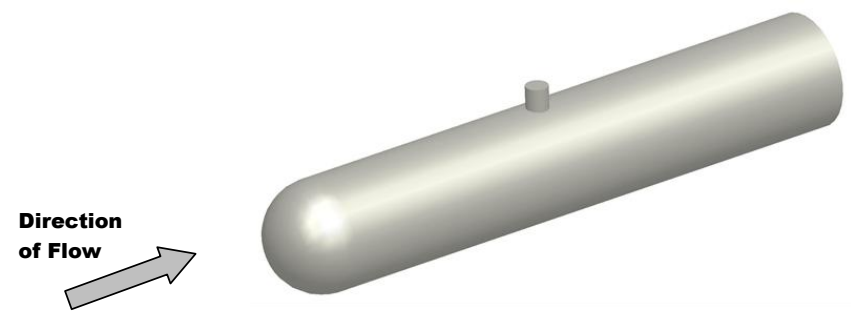

Fig. 1: Solid model of the hull-form with short $\mathrm{H}=\mathrm{D}$ lateral pin-protuberance 
Geometrical features include a clean hemispherical nose having a radius of $20 \mathrm{~mm}$ attached to a cylinder of length of $220.9 \mathrm{~mm}$. The body-fixed reference frame is used, with the origin being placed at the centre line of the nose of the hull-form. Side view of the hull-form as a solid model is shown in Fig. 1. It is assumed that a plane of symmetry exist for the hull-form in the $x-y$ plane. Customary hydrodynamic sign convention and reference axis orientation is used, with an incoming flow in the positive x-direction. Nose-down situation is considered as a negative angle of attack causing negative pitching-moment. Orientation of the axes is shown with geometric details (all dimensions are in $\mathrm{mm}$ ) in Fig. 2.
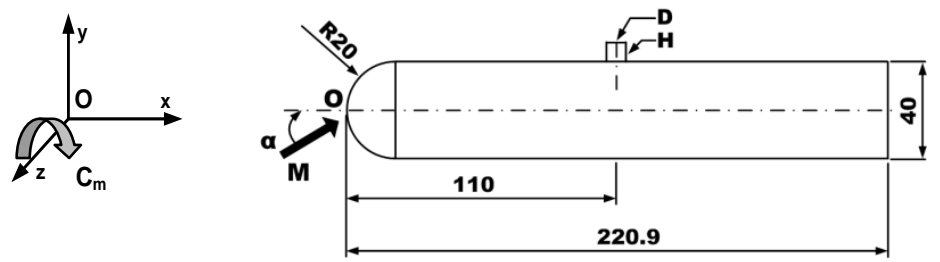

Fig. 2: Hemisphere-cylinder head-form with short $\mathrm{H}=\mathrm{D}$ lateral pin-protuberance (all dimensions are in [mm]).

\subsection{Computational grid and topology}

Quality of computational grid in term of its density and clustering, required for capturing flow details in the entire domain is maintained in this study using grid generation software PAKGRID (1997). Based on previous flow simulations by Zahir et al (2006), a refined mesh distribution was considered, and is briefly discussed here for the hemisphere-cylinder hull-form case only. Grid generation refining sequence is controlled through refining the grids from a coarse grid level to a super-fine grid, ranging from 0.89 to about 1.336 million grid points. Errors of discretization were minimized by recording changes in the result while moving from one grid level to the next, finally the fine-grid with an estimated maximum error of about $0.5 \%$ in the normal force coefficient and $1.6 \%$ in the pitching moment coefficient is selected. Structured half domain grid is used owing to model and flow symmetry. Computational domain selected for all computations is a fine-mesh with approximately 1.22 million grid points. Grid topology with multiblock domains having a $\mathrm{C}$ - and $\mathrm{H}$ grid type combination was considered. Adequate grid clustering was concentrated near the solid walls to ensure a $\mathrm{y}^{+}$value below 2, the first node of the cell closest to the pin and off the solid surfaces was set at $5 \times 10^{-6}$. The overall topologv for the present mesh is shown in Fig. 3.
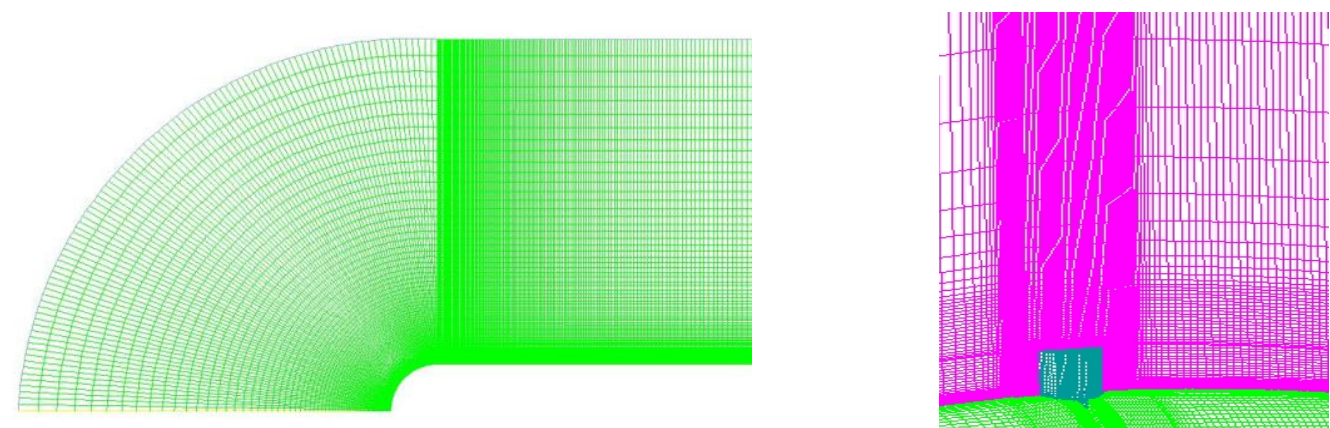

Fig. 3: Body fitted grid-slice of the Hemisphere-cylinder hull-form, with zoomed view of leeward H=D pin.

\section{2 .1 Grid sensitivity study}

In order to assess free mesh solution dependency over the clean axisymmetric hemisphere-cylinder hull-form, four sets of meshes were considered. A reference grid, corresponding to a super-fine mesh topology was used for the initial simulations and was assumed sufficiently refined for accurate flow solution in comparison with the experimental results. The second grid, referred to as a coarse grid was obtained from the reference grid by removing one third of the grid points from all the blocks edges. The third grid, which is termed as a medium grid case was obtained by adding one third of grid points to all the blocks edges. With respect to the fine grid solution, the coarse grid case showed a notable discrepancy while conserving the lift coefficient trend. However, a small or negligible discrepancy was observed between the medium-dense and fine grids solutions. It was concluded that the fine grid was sufficiently dense to compute the flow solutions with reasonable accuracy and used further in the present study.

Using clean hemisphere-cylinder head-form geometry (without pin) and fixing flow conditions as M=0.6 with an angle of attack of zero and 12 degrees, satisfactory convergence is derived by comparing the computed static aerodynamic coefficients with the experimental aerodynamic data [ASAC (2003)]. Table 1 shows the summary of the computed static aerodynamic coefficients along with the mesh size details. 
Table 1: Data summary of mesh qualities for clean hemisphere-cylinder configuration (without pin) at $\mathrm{M}=0.6$.

\begin{tabular}{|c|c|c|c|c|c|}
\hline Comparison & DATA & \multicolumn{3}{|c|}{ MESH QUALITY REFINEMENT LEVELS } \\
\cline { 3 - 6 } Parameter at $\mathbf{M = 0 . 6}$ & [ASAC(2003)] & $\begin{array}{c}\text { COARSE } \\
\mathbf{0 . 8 9} \text { million }\end{array}$ & $\begin{array}{c}\text { MEDIUM } \\
\mathbf{0 . 9 8} \text { million }\end{array}$ & $\begin{array}{c}\text { FINE } \\
\mathbf{1 . 2 2} \text { million }\end{array}$ & $\begin{array}{c}\text { SUPER-FINE } \\
\text { 1.336 million }\end{array}$ \\
\hline $\boldsymbol{C}_{\boldsymbol{Y}}$ at $\boldsymbol{\alpha}=\boldsymbol{0}^{\boldsymbol{0}}$ & 0.0000 & -0.0003 & -0.000201 & -0.00019 & -0.00018 \\
\hline $\boldsymbol{C}_{\boldsymbol{X}}$ at $\boldsymbol{\alpha}=\boldsymbol{0}^{\boldsymbol{0}}$ & 0.23560 & 0.2485971 & 0.2388606 & 0.235678 & 0.2367810 \\
\hline $\boldsymbol{C}_{\boldsymbol{Y}}$ at $\boldsymbol{\alpha}=\mathbf{1 2}^{\mathbf{0}}$ & -0.50349 & -0.528663 & -0.520037 & -0.5070689 & -0.5060168 \\
\hline $\boldsymbol{C}_{\boldsymbol{X}}$ at $\boldsymbol{\alpha}=\mathbf{1 2}^{\boldsymbol{0}}$ & 0.28160 & 0.2995713 & 0.2964203 & 0.281887 & 0.2817476 \\
\hline
\end{tabular}

\subsection{Free stream conditions}

Free stream Mach number was set at $\mathrm{M}=0.5$ to 0.6 at an angle of attack of zero and -12 degree, in air is considered with a specific heat ratio, $\gamma=1.4$, Prandtl number, $\operatorname{Pr}_{t}=0.9$, and Gas constant, $\mathrm{R}=286.7 \mathrm{~J} / \mathrm{kg} \mathrm{K}$. Consequently, the flow conditions taken are; $\mathrm{T}_{\infty}=288 \mathrm{~K}, \mathrm{P}=101.325 \mathrm{kPa}$, for reference simulations, Further, simulations were performed for varying Reynolds number, i.e. $1.5 \times 10^{5}$ and $2.9 \times 10^{5}$ with associated angle of attack increase from a value of 20 to 30 degrees.

Underwater simulations were conducted using different depths in sea by changing ambient pressure. At sea level maximum pressure on the hull-form is $1.426 \times 10^{5} \mathrm{~N} / \mathrm{m}^{2}$ and at $400 \mathrm{~m}$ depth maximum pressure is taken as $4.184 \times 10^{6} \mathrm{~N} / \mathrm{m}^{2}$. For pressure distribution computations, increase in Reynolds number from $\operatorname{Re}=1.5 \times 10^{5}$ to $2.9 \times 10^{5}$ was considered while the change in angle of incidence is taken from zero to 30 degrees.

\subsection{Boundary conditions}

Free stream conditions and turbulence intensity at the inflow are specified, while axisymmetric flowfield is studied with no-slip adiabatic condition applied to all solid surfaces for the nose-cylinder and the pin surfaces. Plane of symmetry was specified as per symmetry boundary conditions and for the degenerated surfaces, coalescence boundary condition was used.

\subsection{Numerical method}

Steady state simulations were performed first for a validation exercise and the evaluation of the clean hemisphere-cylinder hull-form configuration subject to fixed air speed and varying angle of incidences. After the validation exercise, the pin-protuberance attached to the hemisphere-cylinder hull-form geometry was solved for varying flow conditions in air and water. The simulations were performed using the Navier Stokes solver PAK-3D; it is an in-house developed solver, which is based on the finite volume method; employing Jameson's central differencing scheme with artificial dissipation, for convective fluxes of the NS equations. The convective terms of the turbulence transport equations were discretized using a first-order upwind Roe scheme. The discretized NS equations were integrated explicitly in time using multi-step methods while the turbulence equations were solved implicitly using the diagonal dominant alternating direction implicit algorithm. For steady-state solutions, a local time-stepping approach was used to accelerate the convergence. A dimensionless time step of 10-4, with 5 to 40 inner iterations within a time step, was used to achieve an accurate solution. Time-accurate solutions were ensured by a convergence criterion requiring a residual drop of four orders of magnitude, within each time step. The algorithm in general uses a working fluid as an ideal gas. Perfect gas law was used with conventional values of gas constant, $\mathrm{R}$ and specific heat ratio, $\gamma$. Sutherland's viscosity law was used for handling viscosity, while, heat transfer effect due to turbulence are catered by use of constant turbulent Prandtl number. Initial conditions for free stream were specified over complete fluid domain, including, pressure, temperature, Mach number and appropriate angle of attack or side slip angles to initialize. Grid boundaries are kept sufficiently far enough to justify freestream assumption at the boundaries. To investigate incompressible turbulent flow around blunt axisymmetric bodies the algorithm option of pressure/velocity coupled solution was used with single precision and with standard k- $\varepsilon$ turbulence model and with modified wall functions were used in this study.

For cavitation, a multi-phase approach has been applied to model the flow about submerged head forms. The PAK-3D algorithm has an additional option to incorporate multi-phase mixture of constituent volume fraction transport/generation for condensable vapor and non-condensable gas fields treated as a single fluid which allows the phases to interpenetrate, hence mixture momentum and turbulence scalar equations are also solved. Mass transfer modelling provides exchange between mixture vapor phases. By solving separate continuity equations for mixture, one can account for the separate dynamics and thermodynamics of the condensable vapor, and noncondensable gas fields.

Cavitation inception also depends on local turbulence level, so it is a function of Reynolds number. However in present computations, Reynolds number is kept constant while the cavitation number is varied through a change 
in the free stream pressure. Also it is assumed that there is no effect of turbulence level on cavitation inception. Hence the continuity equation for the mixture can be written in the form:

$\frac{\partial}{\partial \mathrm{t}}\left(\rho_{\mathrm{m}}\right)+\nabla \bullet\left(\rho_{\mathrm{m}} \overrightarrow{\mathrm{v}}_{\mathrm{m}}\right)=\stackrel{\bullet}{\mathrm{m}}$

where, $\overrightarrow{\mathrm{v}}_{\mathrm{m}}=\frac{\sum_{\kappa=\mathrm{I}}^{\mathrm{V}} \alpha_{\kappa} \rho_{\kappa} \mathrm{v}_{\kappa}}{\rho_{\mu}}$

and $\rho_{\mathrm{m}}=\sum_{\mathrm{k}=1}^{\mathrm{n}} \alpha_{\mathrm{k}} \rho_{\mathrm{k}}$

The momentum equation for the mixture is obtained by summing the individual momentum equations for all phases. It is expressed as:

$$
\begin{aligned}
\frac{\partial}{\partial \mathrm{t}}\left(\rho_{\mathrm{m}} \overrightarrow{\mathrm{v}}_{\mathrm{m}}\right)+\nabla \bullet\left(\rho_{\mathrm{m}} \overrightarrow{\mathrm{v}}_{\mathrm{m}} \overrightarrow{\mathrm{v}}_{\mathrm{m}}\right)= & -\nabla \mathrm{p}+\nabla \bullet\left[\mu_{\mathrm{m}}\left(\nabla \overrightarrow{\mathrm{v}}_{\mathrm{m}}+\nabla \overrightarrow{\mathrm{v}}_{\mathrm{m}}^{\mathrm{T}}\right)\right]+\rho_{\mathrm{m}} \overrightarrow{\mathrm{g}}+\overline{\mathrm{F}} \\
& +\nabla \bullet\left(\sum_{\mathrm{k}=1}^{\mathrm{n}} \alpha_{\mathrm{k}} \rho_{\mathrm{k}} \overrightarrow{\mathrm{v}}_{\mathrm{dr}, \mathrm{k}} \overrightarrow{\mathrm{v}}_{\mathrm{dr}, \mathrm{k}}\right)
\end{aligned}
$$

Where $\mathrm{n}$ is the number of phase

$$
\begin{aligned}
& \mu_{\mathrm{m}}=\sum_{\mathrm{k}=1}^{\mathrm{n}} \alpha_{\mathrm{k}} \mu_{\mathrm{k}} \\
& \overrightarrow{\mathrm{v}}_{\mathrm{dr}, \mathrm{k}}=\overrightarrow{\mathrm{v}}_{\mathrm{k}}-\overrightarrow{\mathrm{v}}_{\mathrm{m}}
\end{aligned}
$$

volume fraction equation for secondary phase, $\mathrm{p}$ is:

$$
\frac{\partial}{\partial \mathrm{t}}\left(\alpha_{\mathrm{p}} \rho_{\mathrm{p}}\right)+\nabla \bullet\left(\alpha_{\mathrm{p}} \rho_{\mathrm{p}} \overrightarrow{\mathrm{v}}_{\mathrm{m}}\right)=-\nabla \bullet\left(\alpha_{\mathrm{p}} \rho_{\mathrm{p}} \overrightarrow{\mathrm{v}}_{\mathrm{dr}, \mathrm{p}}\right)
$$

Consider a spherical bubble of radius, $\mathrm{R}$ in an infinite domain of liquid whose temperature far from the bubble is assumed to be a simple constant since temperature gradients were eliminated and uniform heating of the liquid due to any internal heat sources or radiation is not considered. On the other hand, pressure is assumed to be a known quantity and treated as a controlled input, regulating the growth or collapse of the bubble. Assuming the volume change of individual bubbles with respect to space and time is denoted by:

$$
\varphi(\overrightarrow{\mathrm{r}}, \mathrm{t})=\frac{4}{3} \pi \mathrm{R}^{3}
$$

The volume fraction of vapor is defined as

$\alpha_{\mathrm{v}}=\frac{\varphi \eta}{1+\varphi \eta}$

Where, $\eta$ is the population number of bubbles per unit volume of the liquid.

The volume fraction of vapor is calculated by:

$$
\frac{\partial}{\partial \mathrm{t}}\left(\alpha_{\mathrm{p}}\right)+\nabla\left(\alpha_{\mathrm{p}} \overrightarrow{\mathrm{v}}_{\mathrm{m}}\right)=\frac{\rho_{1}}{\rho_{\mathrm{m}}} \frac{\eta}{(1+\eta \varphi)^{2}} \frac{\mathrm{d} \varphi}{\mathrm{dt}}+\frac{\alpha \rho_{\mathrm{v}}}{\rho_{\mathrm{m}}} \frac{\mathrm{d} \rho_{\mathrm{m}}}{\mathrm{dt}}
$$

The formation and collapse of a bubble is modeled as a phase transformation, modelling of this process requires details of the thermodynamic behavior of the fluid near a phase transition point and the formation of interfaces. Simplified approach is the use of empirical factors, describing the transformation of liquid to vapor and the transformation of vapor back to liquid. For transformation of liquid to vapor, is modeled as being proportional to the liquid volume fraction and the amount by which the pressure is below the vapor pressure. For transformation a simplified form of the Rayleigh-Plesset equation relates the pressure and the bubble volume, $\varphi$;

$$
\mathbf{R} \frac{\mathbf{d}^{2} \mathbf{R}}{\mathrm{dt}^{2}}+\frac{3}{2}\left(\frac{\mathrm{dR}}{\mathrm{dt}}\right)^{2}=\frac{\mathrm{p}_{\mathrm{B}}-\mathrm{p}}{\rho_{1}}-\frac{2 \sigma}{\rho_{1} \mathbf{R}}-4 \frac{\mu_{1}}{\rho_{1} \mathbf{R}} \frac{\mathrm{dR}}{\mathrm{dt}}
$$

while the process of bubble growth and collapse is given by: 


$$
\frac{\mathrm{dR}}{\mathrm{dt}}= \begin{cases}\sqrt{\frac{2\left(\mathrm{p}_{\mathrm{B}}-\mathrm{p}\right)}{3 \rho_{1}},} & \mathrm{p}_{\mathrm{v}}>\mathrm{p} \\ \sqrt{\frac{2\left(\mathrm{p}_{\mathrm{B}}-\mathrm{p}\right)}{3 \rho_{1}},} & \mathrm{p}_{\mathrm{v}}<\mathrm{p}\end{cases}
$$

On attainment of convergence criteria, information of primitive variables at that iteration level got postprocessed for calculation of non-dimensional axial/ lateral pressure distributions, as well as for all static aerohydrodynamic coefficients. Post-processing software, LOOK is used for pressure, Mach contours and velocity vector plotting.

\section{Results and Discussion}

\subsection{Validation of the numerical solutions}

This section presents the results and discussions based on the numerical simulation of flow at Mach 0.6 over the hemisphere-cylinder hull-form configuration. Clean hemisphere-cylinder geometry was studied with varying angle of attack situations. A general aim of this validation work was to determine the aerodynamic flow characteristics of the hemisphere-cylinder geometry. In the first part of the study, the numerical tool is validated through comparison of (a) zero-incidence drag coefficient, and by (b) computation of axial pressure distribution on the hemisphere-cylinder surface.

\section{1-a. Zero-incidence axial force coefficient}

This section of the study presents results of validation carried out for the simulations made for the clean hemisphere-cylinder hull-form with the incoming subsonic flow of $\mathrm{M}=0.6$ with the experimental data. Accordingly, calculations for aerodynamic normal force (positive in upward direction), and axial force coefficient (positive in axial flow direction) is made (considering cylinder base area as a reference), with the increase in angle of attack. According to sign convention, in case the hull geometry is pitched up (nose-up condition) would give rise to a negative pitching moment (nose-down tendency). From the zero-incidence drag coefficient comparisons, it can be seen that computationally the flow is well resolved with an estimated maximum error of about $0.5 \%$ is in the normal force coefficient, and about $1.2 \%$ in the axial force coefficient, significant increase in normal force and axial force is computed by an increase in angle of attack from zero to 12 degrees; as respective rise in the aerodynamic coefficients is as expected. The experimental drag coefficient data has uncertainties (due to model support arrangement in the wind tunnel and base pressure measurements in presence of sting support). The computed zero-incidence axial force coefficient is observed to have a negligible discrepancy in comparison with the experimental data. Hence, it is concluded that the computed flow solutions are with reasonable accuracy.

Table 2: Computed aerodynamic coefficients of clean hemisphere-cylinder (without pin) at $\mathrm{M}=0.6$.

\begin{tabular}{|c|c|c|c|c|}
\hline \multirow{2}{*}{$\begin{array}{l}\text { Aerodynamic } \\
\text { Coefficients / } \\
\text { Angle of Attack }\end{array}$} & \multicolumn{4}{|c|}{ Mach number } \\
\cline { 2 - 5 } & \multicolumn{2}{|c|}{ [ASAC DATA (2003)] } & \multicolumn{2}{|c|}{ PAK-3D } \\
\hline $\boldsymbol{C}_{\boldsymbol{Y}}$ & $\mathbf{0}^{\mathbf{0}}$ & $\mathbf{1 2}^{\mathbf{0}}$ & $\mathbf{0}^{\mathbf{}}$ & $\mathbf{1 2}^{\mathbf{0}}$ \\
\hline $\boldsymbol{C}_{\boldsymbol{X}}$ & 0.000 & -0.50349 & -0.00019 & -0.5070689 \\
\hline & 0.23560 & 0.28160 & 0.235678 & 0.281887 \\
\hline
\end{tabular}

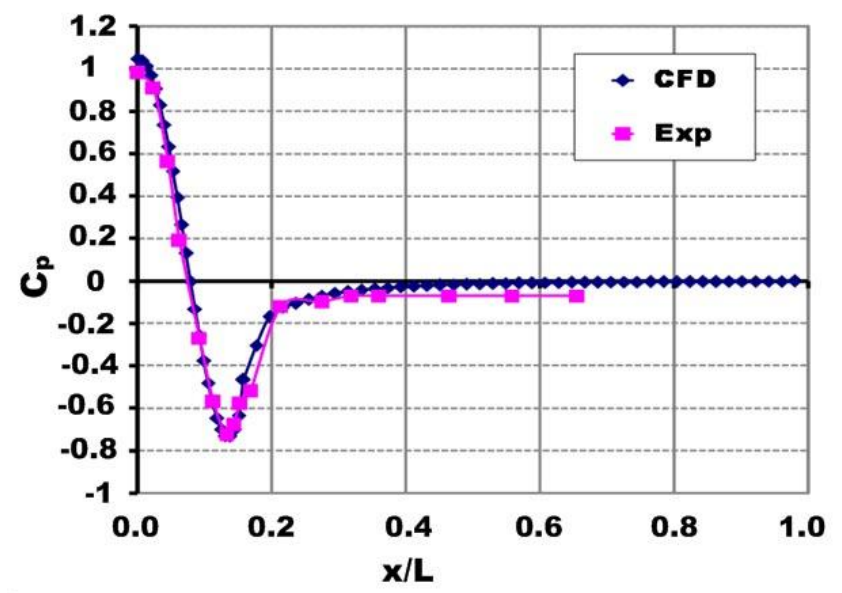

Fig. 4: Computed axial $\mathrm{C}_{\mathrm{p}}$ distribution on clean hemisphere-cylinder head-form, at zero incidence. 


\section{1-b. Axial pressure distribution}

Axial pressure distribution on the surface of a clean hemisphere-cylinder head-form was computed at different Reynolds numbers and by varying incidence angles. For validation purpose, comparison of $C_{p}$ distribution was made with the experimental data, in general the computed solutions matched very well with the experimental data. Maximum error is observed within a tolerance band of $\pm 2 \%$. It is observed that the computed solutions slightly over-predict pressure distribution (at the stagnation point the predicted value of $C_{p}$ is about 1.045). Computed axial $C_{p}$ distribution at $\operatorname{Re}=2.9 \times 10^{5}$ for air as a working fluid is as shown in Fig.4.

\subsection{Computed aerodynamic coefficients}

Further, static aerodynamic coefficients are calculated for blunted hemisphere-cylinder hull-form with pin, at an angle of attack of zero as well as at -12 degrees, with incoming free stream Mach numbers of 0.5 and 0.6. Details are given in Table 3. The coefficient of drag increases from its base value, as calculated at a zero incidence angle; and rises with the increase in angle of attack, for any particular Mach number. Also, in an attempt to evaluate the drag force coefficient, as the sum of the contributions of pressure drag, base drag, skin friction drag and induced drag; an increase in the drag coefficient is observed with the increase in freestream Mach numbers. Generally for clean blunted configuration, pressure drag is usually considered to be the function of Mach number only, while the skin friction drag is a function of Reynolds number.

Table 3: Computed aerodynamic coefficients of hemisphere-cylinder hull-form with H=D pin.

\begin{tabular}{|c|c|c|c|c|}
\hline \multirow{2}{*}{$\begin{array}{c}\text { Aerodynamic } \\
\text { Coefficients / AoA }\end{array}$} & \multicolumn{4}{|c|}{ Mach number } \\
\cline { 2 - 5 } & $\mathbf{0}^{\mathbf{0}}$ & $\mathbf{- 1 2}^{\mathbf{0}}$ & $\mathbf{0}^{\mathbf{o}}$ & $\mathbf{- 1 2}^{\mathbf{0}}$ \\
\hline $\boldsymbol{C}_{\boldsymbol{Y}}$ & $1.494 \times 10^{-2}$ & -0.50617 & $1.521 \times 10^{-2}$ & -0.5035 \\
\hline $\boldsymbol{C}_{\boldsymbol{X}}$ & 0.298086 & 0.30281 & 0.236912 & 0.31035 \\
\hline $\boldsymbol{C}_{\boldsymbol{m}}$ & 0.07732 & -0.68254 & 0.08464 & -0.68654 \\
\hline $\boldsymbol{X}_{\boldsymbol{C} p} / \boldsymbol{L}$ & 0.467816 & 0.47796 & 0.478697 & 0.48673 \\
\hline
\end{tabular}

In comparison with a clean hull-form, hemisphere-cylinder with a pin shows higher axial force coefficient. With the pin, a slight decrease in total drag with an increase in speed is attributed to a negligible contribution of the pin in increasing the overall forebody drag. However an increase in base drag is possibly more dominant causing an overall decrease in total drag. Also, experimental data is often affected by large uncertainties (due to different experimental arrangements of model-sting support in the wind tunnel) in base pressure measurements, only a limited validation study is conducted by comparing the numerical predictions with the experimental data. In addition, general controllability of a hydrodynamic hull-form is based on its longitudinal static stability, which is an important parameter to be calculated for a specific speed and its corresponding incidence angle. It is defined as dimensional difference between the location of the centre of pressure and the location of centre of gravity of the body (measured from the origin at the nose tip). In the present case of the hemisphere-cylinder hull-form with $\mathrm{H}=\mathrm{D}$ pin, the centre of gravity is situated at the cylinder's centre-line, i.e., $X c g=0.085 \mathrm{~m},(X c g / L$ $=0.38479)$. Practically this value is based on the cylinder's geometry and it is the same as achieved in the wind tunnel model design (with some hollow internal sections to accommodate the balance) and also taken as the moment reference centre location in the [ASAC report (2003)]. The non-dimensional centre of pressure location for $M=0.6$, and zero angle of incidence is calculated to be, $X c p / L=0.467816$. This demonstrates that the centre of pressure is aft of the centre of gravity location by a distance of about $0.0083 \mathrm{~m}$, which is termed as the static margin. This location of centre of pressure, i.e., $X c p / L$ tends to move further aft on the hull-form axis from $0.467816 \mathrm{~m}$ to $0.478697 \mathrm{~m}$, as the freestream Mach number is increased from 0.5 to 0.6 . This change provides a supplementary stabilization by an increase in its stability margin, from a value of 0.0083 to a value of 0.00939 . With the introduction of $\mathrm{H}=\mathrm{D}$ pin, for a particular speed further increase in negative angle of attack from zero to -12 degrees, increases in static stability is about $2 \%$. It should be noted that, both the static stability increase and further aft movement of non-dimensional centre of pressure location with the increase in flow Mach number from 0.5 to 0.6 , can be achieved by a fixed $\mathrm{H}=\mathrm{D}$ pin-protuberance. Details of computed static coefficients for the blunted hemisphere-cylinder with $\mathrm{H}=\mathrm{D}$ pin are given in Table 3. Subsequently, the effect of introducing a pin on the hull-form surface creates a flow asymmetry and gives rise to a net normal force. As the pin height increases from 0 to $2 \mathrm{D}$, it causes an additional increase in the normal force coefficient. Variation of static normal force derivative, $C_{Y_{\alpha}}$ shows a steady increase for negative angle of incidence, as shown in Fig. 5. 


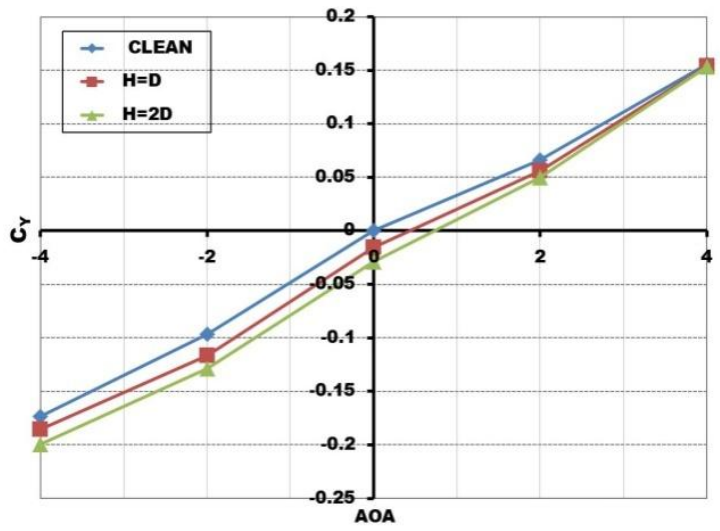

Fig. 5: Plot of $C_{Y}$ with alpha at $\mathrm{M}=0.6$ for clean hull-form, and with pin-height of $\mathrm{H}=\mathrm{D}$ and 2D.

\subsection{Computed axial surface pressure distribution}

The effect of nose geometry on the flow around a head-form is of particular interest in the area of hull-form design for underwater applications. Increase in nose bluntness has hydrodynamic implications, including a general increase in the aero-hydrodynamic axial force coefficient at zero angle of attack. While with an increase in a range of moderate to high angles of attack on a blunt nosed body, a local Werle-Legendre foci-type boundary layer separation, reported by Hsieh (1977) can occur; causing the shear layer to funnel into a spiral vortex emerging tangentially out from the surface before it turned into the main stream to form a longitudinal, horn like, counter rotating primary vortices. Fig. 6 gives a schematic of two flow separation regions that can be observed at low to moderate incidence; i.e., Separation type I - as the usual crossflow separation seen with sharp nosed head forms that results in the formation of leeward longitudinal vortex pairs, and Separation type II which is common to blunt nosed bodies, is also a leeward separation bubble which forms because of the strong adverse pressure gradient resulting from the combination of nose bluntness and incidence. Also, the leeward separation bubble of separation type II tend not to occur at angles of attack less than about 0 degrees, though this is certainly highly Reynolds number dependent, and at moderate angles of attack both separation types I and II can coexist, with type I separation resulting in a closed bubble with a reattachment line. At first, computation of axial pressure distribution is made using water as a working fluid. Computation of local pressure adequately depicts the intensity of peak-pressure and surface pressure distributions for the blunted hull-form at a range of cavitation numbers. For all cases, as the cavitation number is decreased from near a critical value, a cavitation bubble forms and grows.

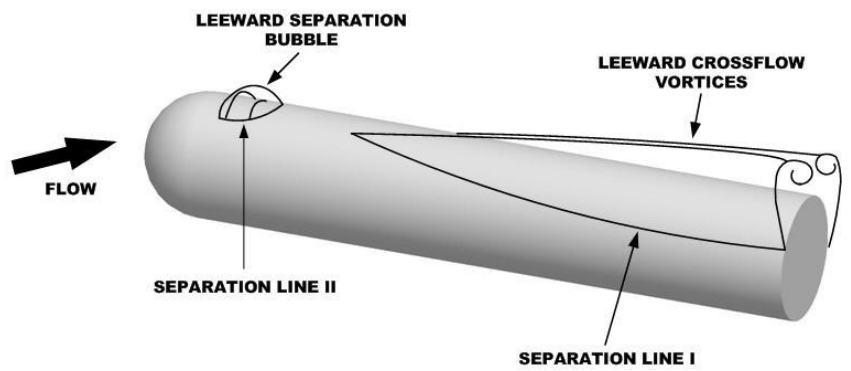

Fig. 6: Schematic flow separation over hemisphere-cylinder hull-form.

Pressure distribution trend negotiates the bubble through a decrease in magnitude, flattening and lengthening of the pressure minimum along the surface. Also, bubble closure initiates an overshoot in pressure recovery due to the local stagnation associated with free stream liquid flowing over the convex curvature at the aft end of the bubble. It is observed that for the hemispherical-cylinder head-form, computations accurately capture the pressure distribution on the hull-form for various Reynolds number, a typically computed surface pressure distribution at sea-level condition is depicted in Fig. 7.

\subsubsection{Computed circumferential pressure distribution}

Study further extended to calculate circumferential pressure distribution on the hemispherical-cylinder hull form at a fixed water depth and varying angle of incidence from zero to 20 and 30 degrees. General aim was to investigate the separated flow pattern over the hull-form at a slightly higher angle of attacks. Occurrence of flow separation by a variation of angle of incidence shows development of flow in cross flow planes along the hullform. At a Reynolds number, $\mathrm{Re}=1.5 \times 10^{5}$ plots of circumferential velocity vectors at a plane normal to the 
plane, positioned at an axial distance of $\mathrm{x} / \mathrm{L}=0.8$ is shown for the case of air and water. Initially, computed results are compared with the vortex formation in air, close agreement was found with the experimental data of Ngoc (1999), as shown in Fig. 8. A small leeward vortex is visible clearly with its core at $\Phi=165$ degrees at an incidence of 20 degrees. With a further increase in the incidence angle from 20 to 30 degrees the numerical computation predicted vortex at a plane situated at $\mathrm{x} / \mathrm{L}=0.8$ shows core location at about $\Phi=140$ degrees while in experimental results separation occurs nearly about 130 degrees. The primary and secondary separation points are found at $\Phi=120$ and 145 degrees, respectively.

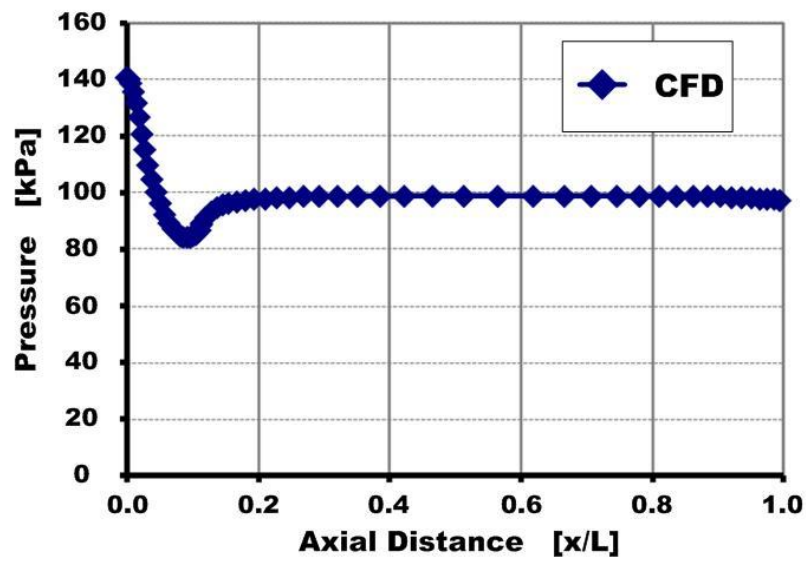

Fig. 7: Computed Leeward Axial pressure distribution at $\alpha=0$ and $\operatorname{Re}=2.9 \times 10^{5}$ for water.
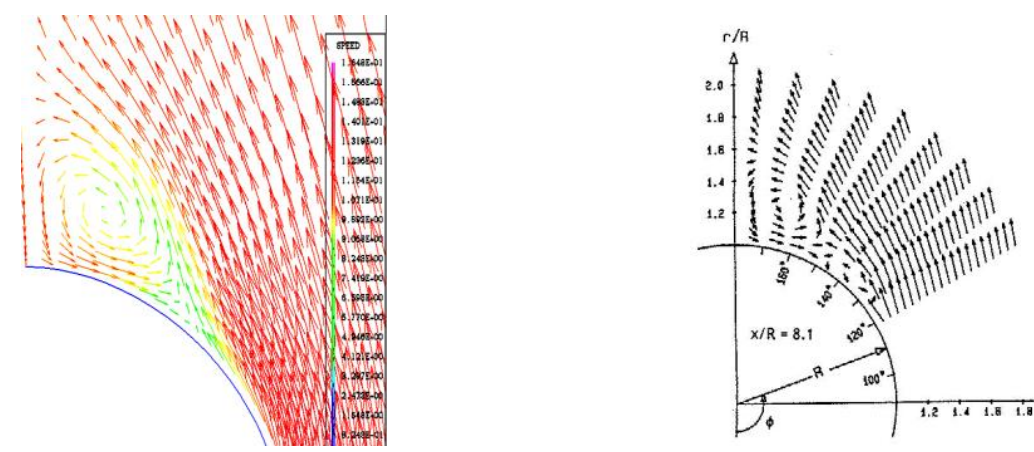

Fig. 8: Velocity vector plots comparison for computed and experimental results at $\mathrm{x} / \mathrm{L}=0.8$ for $\alpha=20 \mathrm{deg}$.
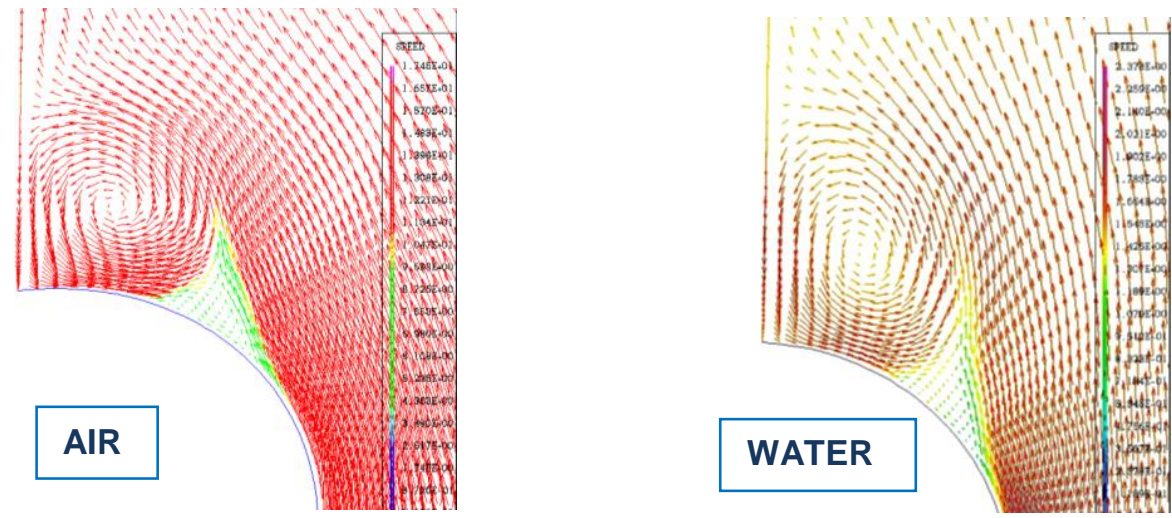

Fig. 9: Velocity vector plots for air and water at $\mathrm{x} / \mathrm{L}=0.8$ for $\alpha=30 \mathrm{deg}$.

The core of the leeward vortex remains at $\Phi=165 \mathrm{deg}$. There exists another smaller vortex (secondary vortex) in the region between the primary and secondary separation points. The sense of rotation of this vortex is the same with the dominant leeward vortex. Velocity vector plots are further made for the Reynolds number $2.9 \mathrm{x}$ $10^{5}$ of the underwater hemisphere-cylinder hull-form at an angle of attack of 30 degrees. The predicted vortex at a transverse plane at a location of $\mathrm{x} / \mathrm{L}=0.8$, occurs with a relatively larger core at about $\Phi=145$ degrees, while the primary and secondary separation points are found at $\Phi=115$ and 135 degrees, respectively. Presence of another small vortex (secondary vortex) is found in the region between the primary and the secondary separation points, with a similar direction of rotation, as of the dominant primary vortex. Comparison of velocity vectors for air and water is shown in Fig. 9.

\subsection{Computed cavitation parameters}

Another significant feature for underwater maneuvering bodies is to study cavitation inception through determination of pressure distribution on the nose-form. The locations where pressure is below saturation vapour pressure are the locations of cavitation inception and the minimum cavitation number at which pressure 
drops below saturation vapor pressure is the cavitation inception number, as mentioned by Liu (1995). For its computation, algorithm selected within the PAK-3D code utilizes a multiphase mixture model with cavitation being implemented based on modelling of bubble growth and its collapse, where cavitation inception is defined as a function of cavitation number $(\sigma)$ given as,

$$
\sigma=\frac{P_{\infty}-P_{V}}{\frac{1}{2} \rho V^{2}}
$$

The fluid in the domain of interest is considered to be mixture of liquid water and water vapor. The density of the bubble nuclei is taken to be $1 \times 10^{6} / \mathrm{m}^{3}$ and the size of the bubble nuclei is of the order of $1 \times 10^{-5} \mathrm{~m}$. Computations are made at $\operatorname{Re}>5 \times 10^{6}$ using $\mathrm{k}-\varepsilon$ turbulence model with non-equilibrium wall function. Distribution of $C_{p}$ on the surface of the head-form is plotted, and as $\left(C_{p}\right)_{\min }<-\sigma$, so the cavitation inception has taken place on the hemispheric nose junction with the cylinder hull-form. Fig. 10 show the vapour phase contours for hemispherical head-form on a very small localized region.
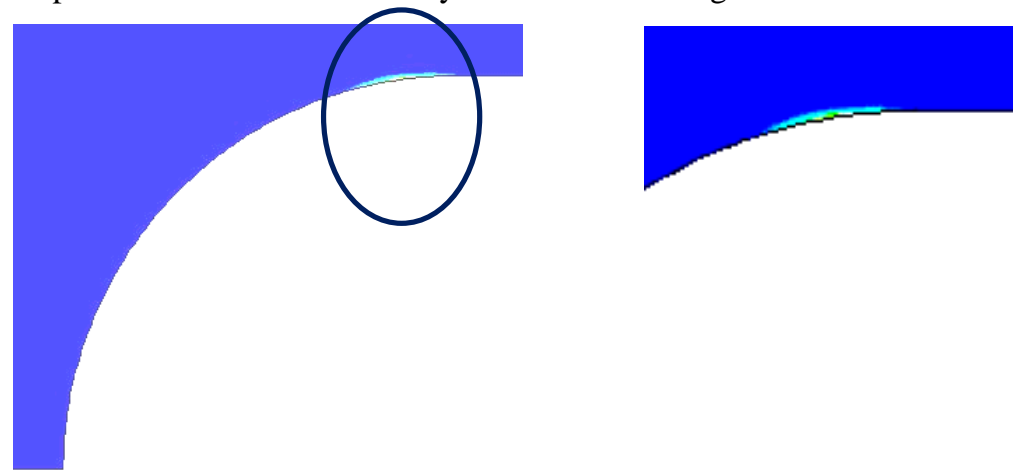

Fig. 10: Vapour phase contours on hemispherical hull-form at zero incidence and with $\sigma=0.70$.

Computations were made for the clean hemispherecylinder hull-form at a fixed velocity of $3.0 \mathrm{~m} / \mathrm{s}$, while a variation of an angle of incidence is considered from 0 to 10 degrees, a maximum variation of the order of $\pm 1 \%$ is observed in comparison with the experimental data of Liu (1999). Computed cavitation inception numbers for different angle of attacks is given in Table 4.

\subsection{Qualitative flow field characterization}

Adequate maps of the flow interactions in presence of the pins fitted to the hemisphere-cylinder hull-form were made, to visualize the flowfield characteristics through a pressure and velocity vector plots. It is observed that when the pin-protuberance height in comparison with the local boundary layer thickness is about 4 to 5 times, the effect of pin-height parameter, $\mathrm{H}$ has a major role in influencing and creating an interaction flowfield. It is found that the chosen pin location, with pin-height $\mathrm{H}=\mathrm{D}$ has a useful effectiveness for the hemisphere-cylinder hull-form for attaining desired flow features conducive of creating a useful net normal force, sufficient to produce pitching moment control. Increase in pin's height causes an increase in the normal force with a corresponding increase in the pitching moment coefficient, generating complex flowfield in vicinity of the pin. Pin's leading edge sees an increase in peak stagnation pressure and a larger flow stagnation region. Causing increase in pin's structural load with extended pin-height and increase in drag force. While in the pin's aft, multi-separated and reattached flow exist with vortex formations. Pressure contours in vicinity of the pin for hull-form at an angle of attack of -2 degrees are as shown in Fig. 11.

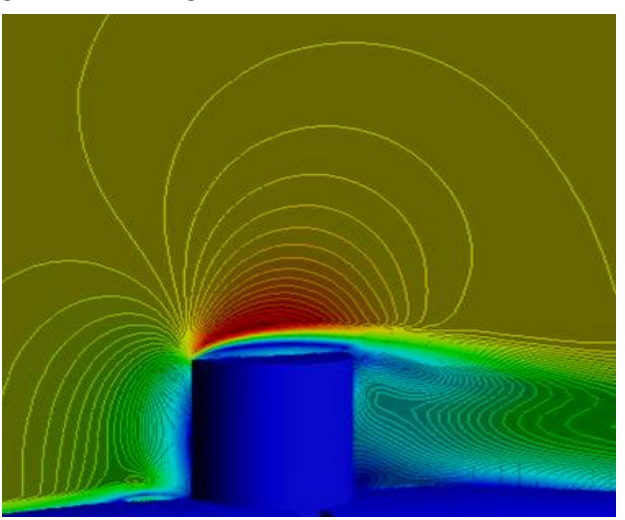

Fig. 11: Pressure contours in vicinity of the $\mathrm{H}=\mathrm{D}$ pin with hull-form at an angle of incidence of -2 degrees.

The numerical solution obtained, in general, found to capture typical velocity variation near pin stagnation and flow separation region, ahead of the pin-protuberance and low pressure zone and flow reattachment in the pin 
aft flow field. Flow field features comparison is made with velocity contours on the plane of symmetry of the pin with the main flow features highlighted.

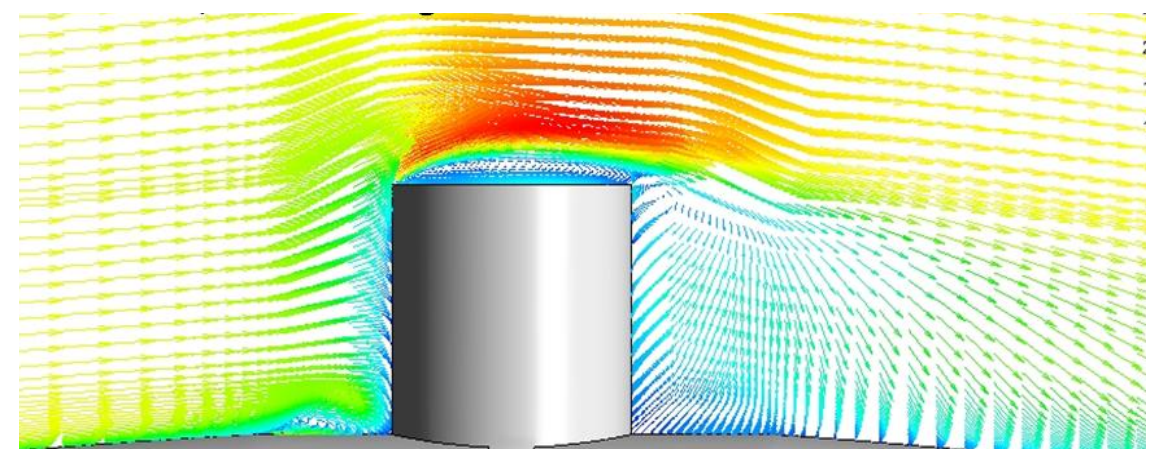

Fig. 12: Velocity vector plots in vicinity of the $\mathrm{H}=\mathrm{D}$ pin at an angle of incidence of -2 degrees.

The vector-plots indicates the recognizable typical flow patterns with stagnation and low pressure zone in vicinity of the $\mathrm{H}=\mathrm{D}$ pin when the hull-form is at -2 degrees incidence, as shown in Fig. 12. Qualitative trend showing the extent of the change in the pressure field in case of hemisphere-cylinder with pin-protuberance height of $H / D=1$, was found effective with a negative incidence angle at Reynold's number $2.9 \times 10^{5}$. Due to flow interaction caused, giving rise to a normal force and subsequent pitching moment, with this altered pressure distribution, a net useable increase in the normal aero-hydrodynamic force is obtained at a submerged depth of $40 \mathrm{~m}$ with non-cavitating and near surface under cavitating conditions.

\section{Conclusions}

A numerical investigation was carried out to explore the utility of a short deployable pin installed on a blunted nose UAV hull-form for its hydrodynamic control and maneuvering. The flow disturbance generated by the pin is studied using CFD techniques at different Reynolds numbers and for fixed pin-height settings, along with quantification of aero-hydrodynamic characteristics. Generally, the validated numerical computations depict the characteristic features of the interaction flowfield produced by the pin-protuberance and the results were found to capture adequately the prominent stagnation peak pressures, separation regions, and low pressure zones.

The study shows that a short pin installed on a hemisphere-cylinder AUV's hull-form, has sufficient control effectiveness to generate a suitable hydrodynamic pitching moment for its pitch control. It is also observed that when the pin-height over the local boundary layer thickness is about a few times, only then it can create an effective interference flowfield. This feature signifies careful choice of pin location in association with the nose shape, bluntness and associated vehicle's speed and attitude. Thus the deployable pin approach is ascertained as a workable idea, promoting the design of a pitch attitude control through continuous adjustment of the pin height. Also, a linear speed control can adjust the speed range for the pre-selected pin-height setting. The two control systems in combination could provide desired maneuvering in a low to intermediate Reynolds numbers and negative angle of attack situations.

\section{Acknowledgements}

The author would like to thank Aerodynamics and Structural Analysis Centre, ASAC, Islamabad, Pakistan; for providing computational and staff support.

\section{References}

Allotta, B., Pugi, L., Costanzi, R., Vettori, G., (2011): Localization algorithm for a fleet of three AUVs by INS, DVL and Range measurements, The 15th Int. Conf. On Adv. Robotics, Tallinn June 20-23.

Alvarez, A., Bertram, V., Gualdesi, L., (2009): Hull hydrodynamic optimization of autonomous underwater vehicles operating at snorkeling depth, Journal of Ocean Engineering. Vol. 36, Issue 1, pp. 105-112.

http://dx.doi.org/10.1016/j.oceaneng.2008.08.006

ASAC Aerodynamics Division, (2003): Aerodynamic Data of Hemisphere-Cylinder Configurations; Vol-2, TRWT-001/03, Aerodynamics and Structural Analysis Centre, Islamabad, Pakistan.

ASAC Aerodynamics Division, (1997): PAK-GRID User's Manual, Aerodynamics and Structural Analysis Centre, Islamabad, Pakistan.

Granville, P.S., (1953): The calculation of the viscous drag of bodies of revolution. Naval Department, The David W. Taylor Model Basin, Washington, Report 849.

https://archive.org/download/calculationofvis00gran/calculationofvis00gran.pdf 
Hackett, J.E., (2000): Drag, lift and pitching moment increments due to wall constraint in a two-dimensional wind tunnel. AIAA 2000-0672, Proceedings of the Thirty Eighth Aerospace Sciences Meeting and Exhibit, Reno, NV. http://arc.aiaa.org/doi/abs/10.2514/6.2000-672

Hsieh, T. (1977): Low supersonic flow over hemisphere cylinder at incidence, AIAA Journal of Spacecraft \& Rockets, Vol. 14., No. 11, pp 662-668. http://arc.aiaa.org/doi/abs/10.2514/3.27990

Jagadeesh, P., Murali, K., (2005): Application of low-Re turbulence models for flow simulations under water vehicle hull forms. JNA\&ME Vol. 2, No. 1.

http://www.banglajol.info/index.php/JNAME/article/download/2029/1889

Li, J., and Lu, C., (2010): Calculation of Added mass of a vehicle running with cavity, J. of Hydrodynamics 2010,22 (3):312-318 vol. 22, No.3. http://www.sciencedirect.com/science/article/pii/S1001605809600603

Kunz, R.F., Boger, D.A., Gibeling, H.J., Govindan, T.R., (1999): A preconditioned navier-stokes method for two-phase flows, AIAA Paper 99-3329, Proc. 14th AIAA CFD Conference.

http://arc.aiaa.org/doi/abs/10.2514/6.1999-3329

Liu, Z., He, H., and Guo, (1995): An experimental study on cavitating axisymmetric head-forms., Ship building of China.

Merkle, C.L., Feng, J., Buelow, P.E.O., (1998): Computational modeling of the dynamics of sheet cavitation, 3rd International Symposium on Cavitation, Grenoble, France.

Nakayama, A., Patel, V.C., (1974): Calculation of the viscous resistance of bodies of revolution. Journal of Hydronautics, Vol. 8 No. 4, pp. 154-162.http://arc.aiaa.org/doi/abs/10.2514/3.48136

Ngoc, T.H., (1999): Hemisphere cylinder at incidence at intermediate to high Reynolds numbers, AIAA Journal Vol. 37, No. 10. http://arc.aiaa.org/doi/abs/10.2514/2.59

Polhamus, E.C., (1957): Effect of Nose Shape on Subsonic Aerodynamic Characteristics of a Body of Revolution Having a Fineness Ratio of 10.94, NACA RM L57F25.

http://naca.central.cranfield.ac.uk/reports/1957/naca-rm-157f25.pdf

Plesset, M.S. and Chapman, R.B. (1971): Collapse of an initially spherical vapor cavity in the neighborhood of a solid boundary. J. Fluid Mechanics, Vol. 47, Issue 2, pp. 283-290.

http://dx.doi.org/10.1017/S0022112071001058

Plesset, M.S. and Prosperetti, A., (1977): Bubble dynamics and cavitation. Ann. Rev. Fluid Mech., 9, 145-185. http://www.annualreviews.org/doi/abs/10.1146/annurev.fl.09.010177.001045

Roddy, R.F., (1990): Investigation of the stability and control characteristics of several configurations of the DARPA SUBOFF Model (DTRC Model 5470) from captive- model experiments. David Taylor Research Centre Report DTRC/SHD-1298-08. http://www.dtic.mil/dtic/tr/fulltext/u2/a227715.pdf

Sung, C.H., Griffin, M.J., Tsai, J.F., Huang, T.T., (1993): Incompressible flow computation of forces and moments on bodies of revolution at incidence. I31st Aerospace Sciences Meeting and Exhibit, January 11-14, AIAA 93-0787. http://arc.aiaa.org/doi/abs/10.2514/6.1993-787

Sung, C.H., Fu, T.C., Griffin, M.J., Huang, T.T., (1995): Validation of incompressible flow computation of forces and moments on axisymmetric bodies at incidence. In: Proceedings of the Thirty Third Aerospace Sciences Meeting and Exhibit, January 9-12, AIAA 95-0528. http://arc.aiaa.org/doi/abs/10.2514/6.1995-528

Ward, L.C., (1978): Force measurements on ten axisymmetric forebodies showing the effects of nose blunting at transonic and supersonic Mach numbers, RAE TR 78093.

Wallskog, H. A. and Hart, R., (1953): Investigation of the drag of blunt-nosed bodies of revolution in free flight mach numbers from 0.6 to 2.3, NACA RM L53D14a.

http://naca.central.cranfield.ac.uk/reports/1953/naca-rm-153d14a.pdf

Xianzhao Y., Yumin S., (2010): Hydrodynamic performance calculation on mini-automatic underwater vehicle, Proceedings of the 2010 IEEE International Conference on Information and Automation June 20 - 23, Harbin, China.

Zahid, Zahir, S., Rafi, (2002): CFD predictions of axial pressure distribution and flow structure of water and air around hemisphere cylinder configuration at intermediate to high Reynolds numbers and at various incidence angles, First International Bhurban Conference on Applied Sciences and Technology.

Zahir, S. and Ye, Z., (2006): Computational Aerodynamic Interaction of a Short Protuberance /Lateral Plate on Blunted Cone Configurations in Hypersonic Flow, AIAA-2006-3172; 24 ${ }^{\text {th }}$ Applied Aerodynamics Conference, CA, USA; 5-8 June.http://arc.aiaa.org/doi/pdf/10.2514/6.2006-3172

Zahir, S., (2011): Aerodynamic Characteristics of a PGMM Shell with Pin-Protuberance under Subsonic Flight Conditions Proceedings of the ICAMS 2011; Islamabad, Pakistan. 